\title{
The Comparison of the Effect of Thoracic Flexibility Exercise and Lumbar Stabilization Exercise on Pain and Heart Rate Variability of Patients with Chronic Low Back Pain
}

\author{
Donghwan Park ${ }^{1}$ and Kang Seong Lee ${ }^{2 *}$ \\ ${ }^{1}$ Department of Physical therapy, Gyeongin Rehabilitation Center Hospital, Korea \\ ${ }^{2 *}$ Department of Biomedical Engineering Welfare Technology, \\ Hanseo University, Korea \\ ${ }^{1}$ donghwan99@naver.com, ${ }^{2}$ cpo114@hanseo.ac.kr
}

\begin{abstract}
To compare the effects of the Thoracic Flexibility Exercise (TFE) with those of Lumbar Stabilization Exercise (LSE) in patients with chronic low back pain. Participants were randomly assigned to TFE $(n=11)$ or LSE $(n=11)$ groups. Chronic low back pain patients in both groups underwent standard physiotherapy for 35 minutes per session. Additionally, TFE and LSE exercises were performed 3 times per week for 4 weeks. The visual analog scale, autonomic nerve activity, and standard deviation normal to normal were measured after 4 weeks of training. After 4 weeks of training, the visual analog scale, autonomic nerve activity, and standard deviation normal to normal improved significantly in both groups after the intervention compared to the baseline $(p<0.05)$. Further, the visual analog scale, autonomic nerve activity, and standard deviation normal to normal improved more significantly in the TFE group than in the LSE group ( $p<0.05)$. This study demonstrates that TFE and LSE are both beneficial for improving pain and heart rate variability. However, TFE was superior to SLE in improving the visual analog scale, autonomic nerve activity, and standard deviation from normal to normal.
\end{abstract}

Keywords: Thoracic flexibility exercise, Lumbar stabilization exercise, Pain, Heart rate variability

\section{Introduction}

Chronic Low Back Pain (CLBP) usually persists for several months, limiting the range of motion of the lower back and lower extremity, and over-activating and controlling the movement of the spinal muscles [1][2]. Also, chronic pain reduces the ability to perform activities of daily living and affects physical problems, including depression and feelings of helplessness, resulting in poor quality of life [3][4]. Heart Rate Variability (HRV) is an indicator of the balance of the sympathetic and parasympathetic nervous systems and is affected by chronic pain, which activates the body's stress response, indicating a reduction in HRV [5][6].

Various interventions, such as the stretching program [7], Lumbar Stabilization Exercise (LSE) [1], and thoracolumbar exercise program [8] had been used to improve balance ability

Article History:

Received (November 29, 2019), Review Result (January 6, 2020), Accepted (February 10, 2020) 
and gait functions in individual patients with CLBP. These training approaches have been shown to effectively improve pain, balance, disability, thoracic mobility, and depression.

Recent studies reported that thoracic flexibility interventions have been applied to reduce back pain, increase spine mobility, and improve balance [8][9][10]. The thoracic curve is affected by the weight load and the type of movement, and the reduction in the motility of the thoracic kyphosis as a result of increased thoracic kyphosis increases the motility as a reward for the lumbar and cervical spine [11][12]. Thoracic Flexibility Exercise (TFE) is a method of spinal exercise that focuses on the extension of the thoracic spine, which results in increased respiration by reducing tension in the intervertebral disc and surrounding tissues [8][9]. Also, TFE reduces the compensatory action of the lumbar spine and improves the mechanical stability of the lumbar spine, which affects pain reduction increased flexibility of the thoracic spine, and balance ability [8][9][13]. Increased thoracic mobility has been shown to balanced muscle development and reduces tension, stress, muscle spasms, and pain [14].

Although TFE is known to stabilize the lumbar spine and improves spinal mobility by focusing on the mobility and rotation of the thoracic spine for CLBP, previous studies have not compared the effects of LSE and TFE in patients with CLBP. The present study's purpose is to compare the effects of a 4-week program of TFE training with those of LSE training on the Visual Analog Scale (VAS) and HRV in patients with CLBP.

\section{Methods}

\subsection{Subjects}

Twenty-two patients with CLBP at Y-Hospital volunteered to participate. The inclusion criteria were: (1) subjects with mechanical low back pain (2) pain lasting more than 3 months (3) no radiation pain under the knee, (4) no sensory loss and reflex loss. (5) Patients with CLBP who were between VAS 3-6 points. The exclusion criteria were: rheumatoid, fractures, spinal tumors, other orthopedic surgery, other chronic pain disorders, major circulatory, respiratory, neurological diseases, heart disease, cognitive deficiency, infection, and other organs. All subjects provided written informed consent for participation and voluntarily signed an experimental agreement. The general characteristics of the participants in this study are shown in [Table 1].

\subsection{Experimental procedures}

A randomized controlled test design was used. The participants were randomized into either the LSE $(n=11)$ or TFE group $(n=11)$ using an online randomization program. All outcome measures and the subjects' characteristics were assessed on study day 1 and 1 day after 4 weeks of exercise, measured by 2 physical therapists who were blinded to the grouping. Specifically, VAS and HRV were the outcome measures. At the start of the trial, participants from both groups were accustomed to LSE or TFE, respectively, depending on randomization. Throughout the trial, all the participants received the standard physiotherapy for 35 minutes per session, and the standard physiotherapy provided was as follows: the first 10 minutes of the hot pack was allocated in the LBP area; the next 20 minutes were spent on transcutaneous electrical nerve stimulation (IN-1200A, Young-in Co. South Korea); the final 5 minutes were spent on ultrasound therapy (ST-10A, Stratek Co. South Korea). Both LSE and TFE were performed in the same place and under the supervision of the main examiner to ensure the proper performance of the main examiner and the safety of all registered participants 3 times per week for 4 weeks. The LSE training consists of three exercise 
methods (bridge exercise, kneeling opposites, and bent-knee leg lift) that can be performed [8][16]. The TFE training consists of three exercise methods (trunk rotation exercise, Mckenzie exercise, and thoracic extension exercise in supine) that can be performed [8][17] All exercises will be maintained for 10 seconds and then returned to the start position. Repeat 10 times after 10 seconds of rest.

Table 1. Clinical information of the patients with chronic low back pain



\subsection{Outcome measurements}

\subsubsection{VAS}

Lumbar pain was assessed using the VAS, which evaluates the perception of pain intensity and consists of a $100 \mathrm{~mm}$ line ranging from "no pain" on the left to "severe pain" on the right. The subjects were asked to point to the current pain level. Higher values indicate more severe pain, and VAS showed good reliability and concurrent validity (ICC .87) [15].

\subsubsection{HRV}

HRV was measured using the autonomic balance tester (SA-3000p, Medi-core Co. Ltd. Korea) for 5 minutes. Autonomic Nerve Activity (ANA) and Standard Deviation Normal to Normal (SDNN) were measured. ANA is less than 90 is bad, $90 \sim 110$ is normal 110 or more is good, SDNN is the standard deviation of the heart rate interval provides information on cardiovascular stability and the ability of the autonomic nervous system to control the body and the higher the value within $30 \sim 60$ the higher the resistance of stress.

\subsection{Data analysis}

PASW Statistics 19 software (SPSS, Chicago, IL, USA) was used for all statistical analyses. Data were presented as mean and standard deviation (SD). Normality was examined using the one-sample Kolmogorov-Smirnov test. Independent t-tests were used to compare between-group means, and paired t-tests were used to compare within-group means. Statistical significance was set at $\mathrm{P}<0.05$.

\section{Results}


Outcome measures are presented in [Table 2]. Between the groups, no significant differences were observed in any of the measured baseline values. All outcome measures showed significant improvement in post-test results compared to pre-test results in both groups $(\mathrm{p}<0.05)$. After treatment, VAS $(\mathrm{p}<0.001)$ was significantly decreased in the TFE group relative to the LSE group. The post-treatment ANA and SDNN were significantly increased in the TFE group as compared with that in the LSE group $(\mathrm{p}<0.001)$.

\section{Discussion}

The present study's purpose is to compare the effect of four-week exercise programs involving LSE and TFE on VAS, ANA, and SDNN in patients with CLBP. Both groups showed significant differences in all outcome measures after the four-week exercise period compared to the baseline. The post-test VAS, ANA, and SDNN were significantly improved in the TFE group compared to the LSE group.

Compared with the LSE group, VAS significantly decreased by $51.2 \%$ in the TFE group after 4 weeks of the study intervention. Furthermore, significant improvements in ankle VAS were observed after treatment, decreasing by $47.5 \%$ in the LSE group and $148.5 \%$ in the TFE group. These results are consistent with those of previous studies [8][9]. Park et al. [8] reported that the application of thoracolumbar exercise improved the static balance, Oswestry disability index, thoracic mobility, and pain compared to the baseline. Heo et al. [9] reported that thoracic mobilization exercise in patients with CLBP improved lumbar stability, balance, and VAS. The TFE reduces the load and compression of the joints of the thoracic and lumbar region through stretching, and decreasing pressure through stretching, increasing the mobility of the thoracic and lumbar region, and improving the mechanical stability of the lumbar spine $[13,18,19]$. These factors likely contribute to the greater decreases in VAS in the TFE group relative to that in the LSE group.

Table 2. Changes in the intervention in each group.

\begin{tabular}{|c|c|c|c|c|c|}
\hline \multirow{2}{*}{ Parameters } & \multicolumn{2}{|c|}{ Lumbar stabilization exercise (n=11) } & \multicolumn{2}{|c|}{ Thoracic flexibility exercise (n=11) } & \multirow{2}{*}{ P } \\
\cline { 2 - 5 } & Pre-test & Post-test & Pre-test & Post-test & \\
\hline VAS (score) & $05.09 \pm 0.83$ & $03.45 \pm 0.82^{*}$ & $05.64 \pm 0.81$ & $02.27 \pm 0.65^{*}$ & $<0.001^{\dagger}$ \\
\hline ANA & $73.91 \pm 7.03$ & $80.45 \pm 8.05^{*}$ & $77.37 \pm 7.02$ & $90.37 \pm 6.82^{*}$ & $0.005^{\dagger}$ \\
\hline SDNN & $28.25 \pm 4.45$ & $33.31 \pm 5.61^{*}$ & $29.48 \pm 4.13$ & $42.41 \pm 5.89 *$ & $<0.001^{\dagger}$ \\
\hline
\end{tabular}

VAS; visual analog scale.

ANA; autonomic nerve activity.

$S D N N$; standard deviation normal to normal.

${ }^{*} p<0.05$ indicate a significant difference between pre-and post-interventions within the group.

$t p<0.05$ indicates a significant difference in the change in scores between the groups.

After the completion of the study interventions, ANA and SDNN were significantly increased by $49.6 \%$ and $60.9 \%$, respectively, in the TFL group as compared with the LSE group. Furthermore, ANA and SDNN after treatment were significantly increased as compared with those at baseline in both groups, particularly by $8.1 \%$ and $15.2 \%$, respectively, in the LSE group. Similar increases were observed in the TFE group, respectively increasing by $14.4 \%$ and $30.5 \%$. These results are consistent with those of previous studies [20][21]. Reis et al. [20] used posteroanterior thoracic mobilization on pain and HRV in patients with 
fibromyalgia and found that a single treatment session with thoracic mobilization therapy improved VAS and HRV. Minarini et al. [21] examined the effects of thoracic spinal manipulation on HRV in asymptomatic patients and found that thoracic spinal manipulation resulted in better HRV activity compared to shame treatment. The TFE reduces the tension in the intervertebral discs and surrounding tissues through the extension of the trunk, resulting in increased respiratory volume along with the extension of the spinal extensor muscles [22]. Also, TFE increases the mobility of the thoracic spine and improves the stability of the lumbar spine leading to decrease pain [9]. Along with the reduction of pain, muscle tension decreased, and increased respiration rate affected the autonomic nervous system of patients with CLBP, indicating an increase in heart rate displacement [4][6]. These factors likely contribute to the greater increases in HRV in the TFE group relative to that in the LSE group.

\section{Conclusion}

Our findings indicate that both TFE and LSE programs improve VAS, ANA, and SDNN. Further, VAS, ANA, and SDNN improved more significantly in the TFE group than in the LSE group. In addition, these findings will contribute to the development of other new therapeutic interventions for patients with CLBP. Future research will need to investigate the effects of TFE on the functional ability, stress, and activities of daily living of patients with CLBP.

\section{References}

[1] T.W. Kang, J. H. Lee, D. H. Park, and H. S. Cynn, "Effect of 6-week lumbar stabilization exercise performed on stable versus unstable surfaces in automobile assembly workers with mechanical chronic low back pain," Work, vol.60, pp.445-454, (2018) DOI: 10.3233/WOR-182743

[2] D. A. MacDonald, A. P. Dawson, and P. W. Hodges, "Behavior of the lumbar multifidus during lower extremity movements in people with recurrent low back pain during symptom remission," journal of orthopedic \& sports physical therapy, vol.41, no.3, pp.155-64, (2011) DOI: 10.2519/jospt.2011.3410

[3] P. Schofield, "Assessment and management of pain in older adults with dementia: a review of current practice and future directions," Current opinion in supportive and palliative care, vol.2, no.2, pp.128-132, (2008) DOI: 10.1097/SPC.0b013e3282ffb406

[4] S. Telles, S. K. Sharma, R. K. Gupta, A. K. Bhardwaj, and A. Balkrishna, "Heart rate variability in chronic low back pain patients randomized to yoga or standard care," BMC complementary and alternative medicine, vol.16, no.1, pp.279-286, (2016) DOI: 10.1186/s12906-016-1271-1

[5] M. E. Berry, I. T. Chapple, J. P. Ginsberg, K. J. Gleichauf, J. A. Meyer, and M.L. Nagpal, "Nonpharmacological intervention for chronic pain in veterans: a pilot study of heart rate variability biofeedback," Global advances in health and medicine, vol.3, no.2 pp.28-33, (2014) DOI: 10.7453/gahmj.2013.075

[6] S. F. Zavarize, M. A. Paschoal, and S. M. Wechsler, "Effects of physiotherapy associated to virtual games in pain perception and heart rate variability in cases of low back pain," Manual Therapy, Posturology \& Rehabilitation Journal Revista Manual Therapy, vol.14, pp.1-7, (2016)

[7] F. R. França, T. N. Burke, R. R. Caffaro, L. A. Ramos, and A. P. Marques, "Effects of muscular stretching and segmental stabilization on functional disability and pain in patients with chronic low back pain: a randomized, controlled trial," Journal of Manipulative and Physiological Therapeutics, vol.35, no.4, pp.27985, (2012) DOI: 10.1016/j.jmpt.2012.04.012

[8] D. H. Park, J. W. Won, J. H. Roh, and E. H. Ko, "Effects of the thoracolumbar exercise program on static standing balance and pain in low back pain patients," Orthopaedic Physical Therapy Practice, vol.24, no.2, pp.78-84, (2012) 
[9] M. Y. Heo, K. Kim, B. Y. Hur, and C. W. Nam, "The effect of lumbar stabilization exercises and thoracic mobilization and exercises on chronic low back pain patients," Journal of physical therapy science, vol.27, no.12, pp.3843-3856, (2015) DOI: 10.1589/jpts.27.3843

[10] N. Handa, H. Yamamoto, T. Tani, T. Kawakami, and R. Takemasa, "The effect of trunk muscle exercises in patients over 40 years of age with chronic low back pain," Journal of orthopedic science, vol.5, no.3, pp.210216, (2000) DOI: 10.1007/s007760050153

[11] S. Edmondston and K. Singer, "Thoracic spine: anatomical and biomechanical considerations for manual therapy," Manual therapy, vol.2, no.3, pp.132-143, DOI: 10.1054/math.1997.0293

[12] M. M. Panjabi, "The stabilizing system of the spine. Part I. Function, dysfunction, adaptation, and enhancement," Journal of spinal disorders, vol.5. pp.383-389

[13] F. R. França, T. N. Burke, E. S. Hanada, and A. P. Marques, "Segmental stabilization and muscular strengthening in chronic low back pain: a comparative study," Clinics, vol.65, no.10, pp.1013-1017, (2010) DOI: 10.1590/S1807-59322010001000015

[14] G. Kim, and O. Kim, "The effect of flexibility training on health-related fitness in healthy adults," J Phys Edu, Vol.39, pp.285-293, (2000)

[15] J. Siebenga, V. Leferink, M. Segers, M. Elzinga, F. Bakker, H. J. ten Duis, P. M. Rommens, and P. Patka, "A prospective cohort study comparing the VAS spine score and Roland-Morris disability questionnaire in patients with a type A traumatic thoracolumbar spinal fracture," European Spine Journal, vol.17, no.8, pp.1096-1100, (2008) DOI: 10.1007/s00586-008-0705-y

[16] H. J. Moon, K. H. Choi, D. H. Kim, H. J. Kim, Y. K. Cho, K. H. Lee, J. H. Kim, and Y. J. Choi, "Effect of lumbar stabilization and dynamic lumbar strengthening exercises in patients with chronic low back pain," Annals of rehabilitation medicine, vol.37, no.1, pp. 110-117, (2013) DOI: 10.5535/arm.2014.38.6.876

[17] R. McKenzie, and P. Van Wijmen, “Treat your own back,” Spinal publications, New Zealand (1985)

[18] L. Lundy-Ekman, "Neuroscience-E-Book: Fundamentals for Rehabilitation," Elsevier Health Sciences St. Louis, Missouri, (2013)

[19] D. W. Vaughn and E. W. Brown, "The influence of an in-home based therapeutic exercise program on thoracic kyphosis angles," Journal of Back and Musculoskeletal Rehabilitation, vol.20, no.4, pp.155-165, (2007)

[20] M. S. Reis, J. L. Q. Durigan, R. Arena, B. R. O. Rossi, R. G. Mendes, and A. Borghi-Silva, "Effects of posteroanterior thoracic mobilization on heart rate variability and pain in women with fibromyalgia," Rehabilitation research and practice, vol.2014, pp.1-6 Article ID 898763, (2014) DOI: 10.1155/2014/898763

[21] G. Minarini, M. Ford, and J. Esteves, "Immediate effect of T2, T5, T11 thoracic spine manipulation of the asymptomatic patient on autonomic nervous system response: Single-blind, parallel-arm controlled-group experiment," International Journal of Osteopathic Medicine, vol.30, pp.12-17, (2018) DOI: 10.1016/j.ijosm.2018.10.002

[22] Y. Yang, Y. H. Kim, Y. K. Kim, J. G. Hur, J. C. Song, and Y. S. Kim, "Effects of thoracic spinal exercise program in VDT workers: pain relief and increased flexibility," Korean Journal of Occupational and Environmental Medicine, vol.16, no.3, pp.250-261, (2004) 